\section{\$9. Accessibility to a Two-Dimensionally Steep Structure of the Electric Field in Tokamak H-mode}

Kasuya, N., Itoh, K.

The electric field plays an important role in H-mode transition. The poloidal structure, in addition to the radial structure, has been pointed out to become steep in the H-mode transport barrier ${ }^{1)}$. The formation of the poloidal electric field induces a radial convective flow by the radial $\mathrm{E} \times \mathrm{B}$ flow, which is candidate to give a new explanation of the rapid $\mathrm{H}$-mode pedestal formation ${ }^{2}$. We have demonstrated the accessibility to the two-dimensionally steep structure by calculating the time evolution of the potential structure on L-H transition ${ }^{3)}$.

Two-dimensional structures of the electrostatic potential, density and flow velocity near the edge of $\mathrm{H}$-mode tokamak plasma are investigated. We have studied the two-dimensional structure in the transport barrier with the model including the nonlinearity in bulk-ion viscosity and turbulence-driven shear viscosity ${ }^{3)}$. The model equation, which describes the poloidal variation as deviation from the flux-surface-averaged quantities, is solved with that describing the radial structure by using the shock ordering ${ }^{1)}$. The following analysis is carried out with the parameter set of the electrode biasing experiment. Electrode biasing is one of the methods for controlling the radial electric field externally, and can induce a transition to an improved confinement state in a controlled manner ${ }^{4)}$.

The time evolution of the two-dimensional structure is calculated in accordance with variation of the applied voltage. The case is shown in Fig. 1 when the applied voltage is ramped up from $200[\mathrm{~V}]$ to $600[\mathrm{~V}]$ in duration of 1 [ms] with the following parameter set: $R=1.75[\mathrm{~m}], a=$ $0.46[\mathrm{~m}], B_{0}=2.35[\mathrm{~T}], T_{\mathrm{i}}=40[\mathrm{eV}], I_{\mathrm{p}}=200[\mathrm{kA}]$ and the boundaries $r=a, a-5[\mathrm{~cm}]$. The electrode current increases and then decreases as the applied voltage increases. The profile of the radial electric field also changes, accordingly. The poloidal flow is given by a $\mathrm{E} \times \mathrm{B}$ flow, so the poloidal Mach number $M_{\mathrm{p}}$ corresponds to the normalized radial electric field. The structural transition of the radial electric field from flat one to solitary one occurs in this case, and the poloidal structure is formed, accordingly. The $\Delta \Phi$ is rather constant at $t=0[\mathrm{~ms}]$, and the perturbation becomes large as the applied voltage increases. At $t=1.6[\mathrm{~ms}]$, the potential perturbation is developed and has $\Delta \Phi_{\max }=50[\mathrm{~V}]$, because $M_{\mathrm{p}}$ becomes large $\left(M_{\mathrm{p}}=1.4\right)$, though $M_{\mathrm{p}}$ has a flat profile. For the duration $t=1.6-5.0[\mathrm{~ms}]$, the radial structure evolves from a flat profile to a solitary profile. The poloidal shock becomes steep in accordance with the radial electric field profile, and the absolute value of the poloidal electric field increases up to $270[\mathrm{~V} / \mathrm{m}]$. The final state $(t=$ $5[\mathrm{~ms}]$ ) has steep gradients both in the radial and poloidal directions, so the accessibility to the two-dimensionally steep structure as obtained in Ref. [2] is confirmed. The typical time scale of the poloidal structural change is given by the poloidal rotation time, which is 100 [ $\mu \mathrm{s}]$ with the parameter set used here, and becomes small with larger ion temperature and smaller device radius $a$. This time scale is shorter than the typical time scale of the radial structural change $\sim 1[\mathrm{~ms}]$. Therefore, a two-dimensional structure evolves in accordance with the radial structural change, which has a longer time scale.

In this way, the existence of the two-dimensionally steep structure in the H-mode transport barrier is confirmed, which shows that the poloidal shock evolves in accordance with the transition of the radial electric field structure, being associated with the radial convective flow. The convective flow velocity is inhomogeneous in radius, and the gradient of the convective flux can be positive in some region. Therefore, sudden increase of derivative of the convective transport at the onset of the transition is candidate to give a new explanation of the rapid $\mathrm{H}$-mode pedestal formation.

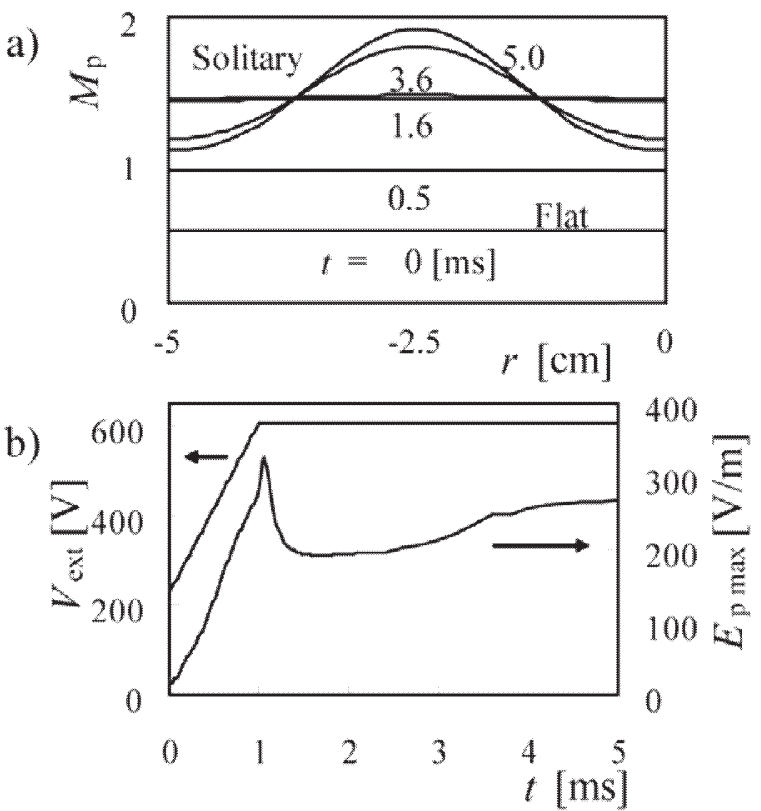

c) Potential profile (poloidal cross section)

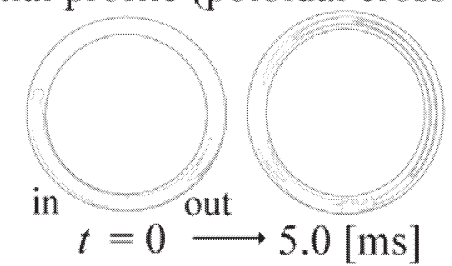

Fig.1: Time evolution of the two-dimensional potential structure in accordance with the applied voltage ramp-up. Time evolutions of (a) the $M_{\mathrm{p}}$ profile, (b) the maximum of $\left|E_{\mathrm{p}}\right|$ and (c) the potential contours on the poloidal cross section are shown.

References

1) Shaing, K. C. et al.: Phys. Fluids B 4 (1992) 404.

2) Kasuya, N. and Itoh, K.: Phys. Rev. Lett. 94 (2005) 195002

3) Kasuya, N. and Itoh, K.: Plasma Phys. Control. Fusion 48 (2006) A319.

4) Weynants, R. R. et al.: Nucl. Fusion 32 (1992) 837. 